\title{
Actualización del tratamiento de las rasopatías
}

\author{
Anna Duat-Rodríguez, Ángela Hernández-Martín
}

Introducción. El término 'rasopatías' agrupa una serie de enfermedades que presentan mutaciones en genes que codifican las proteínas de la vía RAS/MAPK. Estas enfermedades incluyen la neurofibromatosis de tipo 1, el síndrome de Noonan, el síndrome de Legius, el síndrome LEOPARD, el síndrome de Costello y el síndrome cardiofaciocutáneo. La afectación de la vía RAS/MAPK no sólo aumenta la predisposición a desarrollar tumores, sino que también determina la presencia de anomalías fenotípicas y alteraciones en los procesos de aprendizaje.

objetivo. Revisar el papel del uso de estrategias terapéuticas con mecanismos de acción selectivo en las rasopatías.

Desarrollo. El hecho de que la vía RAS participe en un tercio de las neoplasias ha motivado el desarrollo y el estudio de distintos fármacos a este nivel. Algunos de estos fármacos han sido probados en las rasopatías, principalmente en la neurofibromatosis de tipo 1. Analizamos el uso de distintos tratamientos antidiana: fármacos que actúan en los receptores de membrana, como los inhibidores de la tirosincinasa, en la vía mTOR o los inhibidores de MEK. Existe un potencial beneficio de estos últimos en estudios recientes realizados en distintas rasopatías.

Conclusiones. Actualmente, gracias a los resultados de los primeros trabajos desarrollados con inhibidor de MEK basados principalmente en modelos animales, se están realizando múltiples ensayos clínicos prometedores.

Palabras clave. Inhibidor de MEK. mTOR. Neurofibroma plexiforme. Rasopatías. Tratamiento. Vía RAS/MAPK.

\section{Introducción}

Las rasopatías agrupan a un conjunto de enfermedades hereditarias causadas por mutaciones en la línea germinal en los genes que codifican proteínas implicadas en una misma vía metabólica denominada RAS/MAPK (mitogen activated protein kinase).

La vía RAS/MAPK actúa como interruptor de la transducción de señales desde el exterior al interior celular, y activa así una cascada de proteínas implicadas en los procesos de desarrollo celular. La ruta metabólica se inicia cuando el RAS es activado por estímulos extracelulares como el factor de crecimiento que se une al receptor de la tirosincinasa. A este receptor se unen una serie de proteínas intracelulares que reclutan el SOS1 intracitoplasmático, que, a su vez, activa el RAS. El RAS activado promueve la proliferación celular mediante otras vías de señalización. La principal cascada de señalización que continúa a la activación de las proteínas RAS es la vía RAF/MEK/ERK; no obstante, no es la única, y otras vías intracelulares de transducción de la señal pueden ser activadas por las proteínas RAS, como la vía PI3K/Akt/mTOR. Considerando que ambas vías se hiperactivan en las células, puede afectarse más una u otra vía dependiendo del tipo celular, pero las dos están implicadas en la proliferación y la supervivencia celular (Figura) [1,2].

\section{Rasopatías}

La vía RAS/MAPK fue inicialmente estudiada en el contexto de la oncogenia, ya que su desregulación está presente en el 30\% de los cánceres somáticos [3]. A diferencia de las mutaciones somáticas de la vía RAS, cuyo potencial de malignidad es muy elevado, las mutaciones en la línea germinal provocan fundamentalmente anomalías en el desarrollo del individuo, que si bien dependen de forma específica del gen afectado, a menudo se superponen clínicamente. La implicación en la vía RAS/MAPK no sólo aumenta la predisposición a desarrollar tumores, sino que también determina la presencia de anomalías fenotípicas y alteraciones en los procesos de aprendizaje, características que comparten las enfermedades causadas por la mutación de la línea germinal en los genes que codifican proteínas implicadas en la misma vía metabólica. Todas estas enfermedades se conocen con el nombre de rasopatías, y agrupan síndromes fenotípica y etiopatogénicamente similares. Afectan a 1 de cada 1.000 individuos, e incluyen la neurofibromatosis de tipo 1 (NF1), el síndrome de Noonan, el síndrome LEOPARD, el síndrome de Costello, el síndrome cardiofaciocutáneo y el síndrome de Legius [4]. Los pacientes afectos, aunque presentan una enfermedad distinta, comparten un grado variable de dificulta-
Sección de Neurología (A. DuatRodríguez). Servicio de Dermatología (A. Hernández-Martín). Hospital Infantil Universitario Niño Jesús. Madrid, España.

Correspondencia:

Dra. Anna Duat Rodríguez. Sección de Neurología. Hospital Infantil Universitario Niño Jesús. Avda. Menéndez Pelayo, 65 E-28009 Madrid.

E-mail:

anna.duat@salud.madrid.org

Aceptado:

10.04.17.

Cómo citar este artículo: Duat-Rodríguez A, Hernández Martín A. Actualización del tratamiento de las rasopatías. Rev Neurol 2017; 64 (Supl 3): S13-7.

(c) 2017 Revista de Neurología 
Figura. Vía metabólica RAS/MAPK.

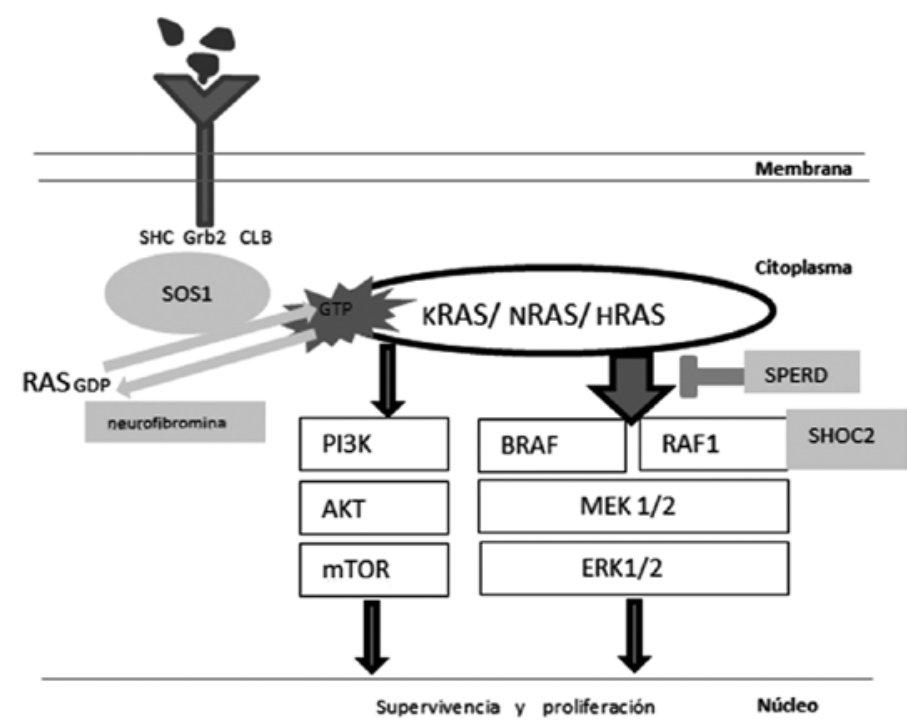

des de aprendizaje, trastornos cardíacos, dismorfismo facial, macrocefalia, talla baja, anomalías cutáneas y, en algunos casos, predisposición al cáncer. Las rasopatías se caracterizan, además de por un solapamiento clínico, por una heterogeneidad genética (Tabla). Algunas las causa un solo gen mutado (NF1, y síndromes de Legius y de Costello); sin embargo, otras se asocian con mutaciones en varios genes (síndromes de Noonan, LEOPARD y cardiofaciocutáneo), e incluso distintas entidades pueden estar causadas por un mismo gen (síndromes de Noonan y LEOPARD). Adicionalmente, existen otras enfermedades relacionadas con mutaciones germinales en la vía RAS/MAPK, como el síndrome de la malformación de capilares-malformación arteriovenosa, el síndrome linfoproliferativo autoinmune o la hiperplasia fibrosa gingival de tipo 1 [5]. Y se continúan describiendo nuevos genes implicados en la vía RAS/MAPK en pacientes con rasgos fenotípicos sugestivos de rasopatías, pero sin mutación en ninguno de los genes conocidos actualmente [6,7].

\section{Fármacos antidiana}

El conocimiento creciente en la última década de las rasopatías, implicadas en una vía cada vez mejor definida, ha motivado los esfuerzos a encontrar un fármaco inhibidor de ésta. Coincide con el desarrollo de nuevos fármacos antineoplásicos, los llama- dos 'antidiana' (targeted therapies). Se diferencian de la quimioterapia porque están diseñados para bloquear de forma específica un aspecto concreto del proceso canceroso, y pueden actuar a distintos niveles. Tienen un mecanismo de acción selectivo basado en los procesos básicos de la biología molecular. Cuando se une un ligando al receptor de membrana situado extracelularmente, se produce un cambio químico en el dominio intracelular del receptor que, a su vez, conlleva una sucesión de interacciones (cascada de señalización) que llegan al núcleo celular para activar la reproducción de la célula. Este proceso básico en la biología celular está inadecuadamente activado en las células tumorales, y se reproduce continuamente. A la vez, produce sustancias proangiogenia para poder nutrirse y continuar proliferando el tumor [8].

Los tratamientos antidiana están dirigidos a uno o varios lugares, y se clasifican según dónde actúen de forma preferente. Pueden actuar uniéndose a los receptores de membrana, como los anticuerpos monoclonales contra receptores de membrana (trastuzumab, cetuximab); en la parte intracelular de los receptores de membrana, como los inhibidores de la tirosincinasa (erlotinib, imatinib, sunitinib, sorafenib); en las vías de señalización, como los inhibidores de la vía mTor (everolimús, sirolimús) o los inhibidores de la vía MEK (trametinib, selumetinib); en la neoangiogenia, o como agentes hormonales o inmunomoduladores [8]. Lamentablemente, los esfuerzos iniciales encaminados a crear un nuevo fármaco inhibidor de la vía RAS/MAPK no tuvieron éxito, aunque nuevos conocimientos y estrategias combinadas resultan más esperanzadores [3].

En las rasopatías, muchos de estos tratamientos han sido probados en relación con la NF1, probablemente por su frecuencia (1 de cada 2.500-3.000 nacidos vivos) y su predisposición a tumores de toda clase. Principalmente se han realizado estudios en neurofibromas plexiformes [9]. Este tipo de neurofibroma está presente en un $23 \%$ en series infantiles [9], y puede tener distinta sintomatología dependiendo de la localización y el tamaño. Pero además, un 8-12\% de los pacientes desarrollarán un tumor maligno de la vaina nerviosa periférica, generalmente en la zona del neurofibroma plexifome. También hay estudios en los gliomas de vía óptica.

En las rasopatías se encontraron resultados desalentadores con fármacos dirigidos. Así, el tipifarnib (inhibidor del RAS), a pesar de ser bien tolerado, no obtuvo los resultados deseados en niños y adultos con neurofibromas plexiformes [10,11]. Tampoco el sorafenib, que actúa en la membrana y el endotelio como inhibidor de VEGFR, c-kit y RAF, 
consiguió la contracción tumoral en niños con neurofibromas plexiformes [12], a pesar de haber conseguido algunos resultados positivos en ratones [13]. Incluso en 11 niños con gliomas de bajo grado, el sorafenib produjo aceleración inesperada y sin precedentes del crecimiento tumoral [14].

Sin embargo, se han conseguido mejores resultados en pacientes con neurofibromas plexiformes actuando en la célula inmunitaria y los neovasos con interferón $\alpha$-2b [15] o con imatinib (inhibidor de la tirosincinasa y c-kit) [16] en estudios en fase 2. También se han ensayado fármacos que actúan en la vía mTOR, dada la activación demostrada de esta vía, y han mostrado resultados variables [17]. Tras buenos resultados con el everolimús en animales con tumor maligno de la vaina nerviosa periférica [18], estudios posteriores muestran resultados inconstantes [13]. El sirolimús, en pacientes con neurofibromas plexiformes en estudios en fase 2, prolonga el tiempo de progresión de neurofibromas plexiformes progresivos [19] y mantiene estables, pero sin mejoría, los no progresivos [20].

Nuevas estrategias para alcanzar dianas moleculares de la vías RAS/MAPK están en estudio a partir del análisis genómico de la expresión de genes en neurofibromas plexiformes y tumor maligno de la vaina nerviosa periférica, tanto humanos como desarrollados en modelos de NF1 en ratones, lo que realizaron Jessen et al, quienes observaron que un inhibidor de MEK, PD0325901, redujo las células que proliferan aberrantemente en los neurofibromas plexiformes y los tumores malignos de la vaina del nervio periférico, y consiguieron prolongar la supervivencia [21]. También Chang et al lograron buenos resultados en ratones con NF1 con leucemia mielomonocítica juvenil [22]. Estudios más recientes obtienen resultados esperanzadores en los neurofibromas plexiformes [23,24] y los tumores malignos de la vaina del nervio periférico usando inhibidores de MEK [23-25], y resultan también exitosos en la restauración ósea de la pseudoartrosis [26].

A pesar de ser menos numerosos, se han publicado algunos trabajos en otras rasopatías. Así, en el síndrome de Noonan se está evaluando el potencial beneficio de inhibidores de MEK [27-29] e inhibidores de la tirosincinasa [30] en el fenotipo y la cardiopatía de ratones. Resultados similares se obtienen en el síndrome cardiofaciocutáneo [31,32].

Las dificultades de aprendizaje y déficit de atención se han estudiado ampliamente en los pacientes con NF1 y en los pacientes con síndrome de Noonan, intentando encontrar nuevos fármacos que mejoren su desarrollo cognitivo basándose en las alteraciones elevadas de la actividad RAS, con aumento
Tabla. Principales síndromes genéticos de la vía RAS/MAPK.

\begin{tabular}{|c|c|c|c|c|}
\hline & Gen & Cromosoma & Proteína & Fenotipo \\
\hline $\begin{array}{l}\text { Neurofibromatosis } \\
\text { de tipo } 1\end{array}$ & $N F 1$ & $17 q 11.2$ & $\begin{array}{l}\text { Neurofi- } \\
\text { bromina }\end{array}$ & $\begin{array}{l}\text { Manchas café con leche y efélides } \\
\text { Nódulos de Lisch } \\
\text { Neurofibromas } \\
\text { Glioma de la vía óptica } \\
\text { Displasia ósea } \\
\text { Dificultades de aprendizaje } \\
\text { Predisposición a desarrollar otros tumores }\end{array}$ \\
\hline Síndrome de Noonan & $\begin{array}{l}\text { PTPN11 } \\
\text { SOS1 } \\
\text { RAF1 } \\
\text { KRAS } \\
\text { NRAS } \\
\text { SHOC2 } \\
\text { CLB }\end{array}$ & $\begin{array}{l}12 q 24.1 \\
2 \mathrm{p} 22.1 \\
3 \mathrm{p} 25.1 \\
12 \mathrm{p} 12.1 \\
1 \mathrm{p} 13.2 \\
10 \mathrm{q} 25.2 \\
11 \mathrm{q} 23.3\end{array}$ & $\begin{array}{l}\text { SHP2 } \\
\text { SOS1 } \\
\text { CRAF } \\
\text { KRAS } \\
\text { NRAS } \\
\text { SHOC2 } \\
\text { CBL }\end{array}$ & $\begin{array}{l}\text { Rasgos faciales característicos } \\
\text { Talla baja } \\
\text { Cardiopatía (estenosis pulmonar, } \\
\text { miocardiopatía hipertrófica) } \\
\text { Criptorquidia } \\
\text { Anomalías linfáticas } \\
\text { Alteraciones sanguíneas }\end{array}$ \\
\hline $\begin{array}{l}\text { Síndrome LEOPARD } \\
\text { (síndrome de Noonan } \\
\text { con múltiples lentigos) }\end{array}$ & $\begin{array}{l}\text { PTPN11 } \\
\text { RAF1 }\end{array}$ & $\begin{array}{l}12 q 24.1 \\
3 p 25.1\end{array}$ & $\begin{array}{c}\text { SHP2 } \\
\text { RAF1/CRAF }\end{array}$ & $\begin{array}{l}\text { Lentigos } \\
\text { Anomalías electrocardiográficas } \\
\text { Hipertelorismo ocular } \\
\text { Estenosis pulmonar } \\
\text { Anomalías genitales } \\
\text { Retraso del crecimiento } \\
\text { Sordera neurosensorial }\end{array}$ \\
\hline
\end{tabular}

Síndrome de Costello HRAS $11 \mathrm{p} 15.5 \quad$ HRAS Déficit cognitivo

Piel laxa con pliegues palmares profundos Lesiones papilomatosas periorificiales Cardiopatía (estenosis pulmonar) Anomalías oftalmológicas Predisposición a desarrollar tumores sólidos (rabdomiosarcoma) Rasgos faciales característicos

\begin{tabular}{|c|c|c|c|c|}
\hline $\begin{array}{l}\text { Síndrome } \\
\text { cardiofaciocutáneo }\end{array}$ & $\begin{array}{c}\text { BRAF } \\
\text { MAP2K1 } \\
\text { MAP2K2 } \\
\text { KRAS }\end{array}$ & $\begin{array}{c}7 q 34 \\
15 q 22.31 \\
19 p 13.3 \\
12 p 12.1\end{array}$ & $\begin{array}{l}\text { BRAF } \\
\text { MEK1 } \\
\text { MEK2 } \\
\text { KRAS }\end{array}$ & $\begin{array}{l}\text { Déficit cognitivo } \\
\text { Cardiopatía } \\
\text { Pelo rizado y escaso } \\
\text { Descamación ictiosiforme, } \\
\text { uleritema ofriógeno, nevus } \\
\text { Anomalías oftalmológicas } \\
\text { Rasgos faciales característicos }\end{array}$ \\
\hline Síndrome de Legius & SPERD1 & $15 q 14$ & SPERD1 & $\begin{array}{l}\text { Manchas café con leche y efélides } \\
\text { Dificultades de aprendizaje }\end{array}$ \\
\hline
\end{tabular}

de la actividad dependiente del ácido gamma-aminobutírico y plasticidad sináptica reducida [33]. Payne et al demuestran la no eficacia de la lovastatina en un estudio controlado con placebo [34]. En la actualidad hay un estudio en curso con lamotrigina en el desarrollo cognitivo de los niños con NF1 tras la demostración reciente de la función atenuada del canal HCN1 (hyperpolarization-activated cyclic nucleotide-gated) [35].

En la actualidad existen múltiples estudios activos, principalmente relacionados con inhibidores del 
MEK en la NF1. Se considera, además, que los resultados pueden ser aún más prometedores con la combinación de diferentes fármacos que actúen en distintas dianas $[17,18,25,36]$. En las rasopatías se han obtenido algunos resultados con dosis más bajas respecto a otros estudios realizados con fármacos antineoplásicos [30]. Y en el caso de los neurofibromas plexiformes, hay que tener presente que es un tipo de tumores de lento crecimiento y en general bien tolerados, y se observa un menor umbral para soportar efectos secundarios $[12,16]$ y resultados desiguales según el paciente y según el neurofibroma $[15,16]$. Es importante encontrar la dosis mínima eficaz.

\section{Conclusiones}

En la actualidad, se están diseñando nuevas estrategias terapéuticas antineoplásicas con mecanismos de acción selectivos, con la posibilidad de combinarse entre ellas o con otras terapias. El hecho de que la vía RAS participe en un tercio de las neoplasias ha motivado el desarrollo y estudio de distintos fármacos en esa vía. El término 'rasopatías' agrupa una serie de enfermedades que presentan mutaciones en genes que codifican las proteínas de la vía RAS. No parece quimérico pensar que fármacos selectivos para esta vía metabólica puedan mejorar estas enfermedades. Actualmente, gracias a los resultados de los primeros trabajos desarrollados con inhibidor de MEK basados principalmente en modelos animales, se están realizando múltiples ensayos clínicos prometedores.

\section{Bibliografía}

1. Niemeyer CM. RAS diseases in children. Haematologica 2014; 99: 1653-62.

2. Hernández-Martín A, Torrelo A. Rasopathies: developmental disorders that predispose to cancer and skin manifestations. Actas Dermosifiliogr 2011; 102: 402-16.

3. Keeton AB, Salter EA, Piazza GA. The RAS-effector interaction as a drug target. Cancer Res 2017; 77: 221-6.

4. Rauen KA. The RASopathies. Annu Rev Genomics Hum Genet 2013; 14: 355-69.

5. Pevec U, Rozman N, Gorsek B, Kunej T. RASopathies: presentation at the genome, interactome, and phenome levels. Mol Syndromol 2016; 7: 72-9.

6. Tidyman WE, Rauen KA. Expansion of the RASopathies. Curr Genet Med Rep 2016; 4: 57-64.

7. Nowaczyk MJ, Thompson BA, Zeesman S, Moog U, SánchezLara PA, Magoulas PL, et al. Deletion of MAP2K2/MEK2: a novel mechanism for a RASopathy? Clin Genet 2014; 85: 138-46.

8. Grau JJ, Caballero M, Tagliapetra A. New antitarget antineoplastic drugs. Clinical indications in the treatment of cancer. Med Clin (Barc) 2011; 137: 370-6.

9. Duat-Rodríguez A, Martos-Moreno GA, Martín SantoDomingo Y, Hernández-Martín A, Espejo-Saavedra Roca JM, Ruiz-Falcó Rojas ML, et al. Phenotypic and genetic features in neurofibromatosis type 1 in children. An Pediatr (Barc) 2015; 83: 173-82.

10. Widemann BC, Babovic-Vuksanovic D, Dombi E, Wolters PL Goldman S, Martin S, et al. Phase II trial of pirfenidone in children and young adults with neurofibromatosis type 1 and progressive plexiform neurofibromas. Pediatr Blood Cancer 2014; 61: 1598-602.

11. Widemann BC, Dombi E, Gillespie A, Wolters PL, Belasco J, Goldman S, et al. Phase 2 randomized, flexible crossover, double-blinded, placebo-controlled trial of the farnesyltransferase inhibitor tipifarnib in children and young adults with neurofibromatosis type 1 and progressive plexiform neurofibromas. Neuro Oncol 2014; 16: 707-18.

12. Kim A, Dombi E, Tepas K, Fox E, Martin S, Wolters P, et al. Phase I trial and pharmacokinetic study of sorafenib in children with neurofibromatosis type I and plexiform neurofibromas. Pediatr Blood Cancer 2013; 60: 396-401.

13. Wu J, Dombi E, Jousma E, Scott Dunn R, Lindquist D, Schnell BM, et al. Preclinical testing of sorafenib and RAD001 in the $\mathrm{Nf}$ (flox/flox); DhhCre mouse model of plexiform neurofibroma using magnetic resonance imaging. Pediatr Blood Cancer 2012; 58: 173-80.

14. Karajannis MA, Legault G, Fisher MJ, Milla SS, Cohen KJ, Wisoff JH, et al. Phase II study of sorafenib in children with recurrent or progressive low-grade astrocytomas. Neuro Oncol 2014; 16: 1408-16.

15. Jakacki RI, Dombi E, Steinberg SM, Goldman S, Kieran MW, Ullrich NJ, et al. Phase II trial of pegylated interferon alfa- $2 b$ in young patients with neurofibromatosis type 1 and unresectable plexiform neurofibromas. Neuro Oncol 2017; 19: 289-97.

16. Robertson KA, Nalepa G, Yang FC, Bowers DC, Ho CY, Hutchins GD, et al. Imatinib mesylate for plexiform neurofibromas in patients with neurofibromatosis type 1: a phase 2 trial. Lancet Oncol 2012; 13: 1218-24.

17. Endo M, Yamamoto H, Setsu N, Kohashi K, Takahashi Y, Ishii T, et al. Prognostic significance of AKT/mTOR and MAPK pathways and antitumor effect of mTOR inhibitor in NF1-related and sporadic malignant peripheral nerve sheath tumors. Clin Cancer Res 2013; 19: 450-61.

18. Johansson G, Mahller YY, Collins MH, Kim MO, Nobukuni T, Perentesis J, et al. Effective in vivo targeting of the mammalian target of rapamycin pathway in malignant peripheral nerve sheath tumors. Mol Cancer Ther 2008; 7: 1237-45.

19. Weiss B, Widemann BC, Wolters P, Dombi E, Vinks A, Cantor A, et al. Sirolimus for progressive neurofibromatosis type 1-associated plexiform neurofibromas: a neurofibromatosis Clinical Trials Consortium phase II study. Neuro Oncol 2015; 17: 596-603.

20. Weiss B, Widemann BC, Wolters P, Dombi E, Vinks AA, Cantor A, et al. Sirolimus for non-progressive NF1-associated plexiform neurofibromas: an NF clinical trials consortium phase II study. Pediatr Blood Cancer 2014; 61: 982-6.

21. Jessen WJ, Miller SJ, Jousma E, Wu J, Rizvi TA, Brundage ME, et al. MEK inhibition exhibits efficacy in human and mouse neurofibromatosis tumors. J Clin Invest 2013; 123: 340-7.

22. Chang T, Krisman K, Theobald EH, Xu J, Akutagawa J, Lauchle JO, et al. Sustained MEK inhibition abrogates myeloproliferative disease in Nf1 mutant mice. J Clin Invest 2013; 123: 335-9.

23. Jousma E, Rizvi TA, Wu J, Janhofer D, Dombi E, Dunn RS, et al. Preclinical assessments of the MEK inhibitor PD-0325901 in a mouse model of Neurofibromatosis type 1. Pediatr Blood Cancer 2015; 62: 1709-16.

24. Dombi E, Baldwin A, Marcus LJ, Fisher MJ, Weiss B, Kim A, et al. Activity of selumetinib in neurofibromatosis type 1-related plexiform neurofibromas. N Engl J Med 2016; 375: 2550-60.

25. Ahsan S, Ge Y, Tainsky MA. Combinatorial therapeutic targeting of BMP2 and MEK-ERK pathways in NF1-associated malignant peripheral nerve sheath tumors. Oncotarget 2016; 7: 57171-85.

26. De la Croix Ndong J, Stevens DM, Vignaux G, Uppuganti S, Perrien DS, Yang X, et al. Combined MEK inhibition and BMP2 treatment promotes osteoblast differentiation and bone healing in Nf1Osx -/- mice. J Bone Miner Res 2015; 30: 55-63. 
27. Hernández-Porras I, Fabbiano S, Schuhmacher AJ, Aicher A, Canamero M, Camara JA, et al. K-RasV14I recapitulates Noonan syndrome in mice. Proc Natl Acad Sci U S A 2014; 111: $16395-400$.

28. Wu X, Simpson J, Hong JH, Kim KH, Thavarajah NK, Backx PH, et al. MEK-ERK pathway modulation ameliorates disease phenotypes in a mouse model of Noonan syndrome associated with the Raf1(L613V) mutation. J Clin Invest 2011; 121: 1009-25.

29. Stevenson DA, Schill L, Schoyer L, Andresen BS, Bakker A, Bayrak-Toydemir P, et al. The Fourth International Symposium on Genetic Disorders of the Ras/MAPK pathway. Am J Med Genet A 2016; 170: 1959-66.

30. Yi JS, Huang Y, Kwaczala AT, Kuo IY, Ehrlich BE, Campbell SG, et al. Low-dose dasatinib rescues cardiac function in Noonan syndrome. JCI Insight 2016; 1: e90220.

31. Inoue S, Moriya M, Watanabe Y, Miyagawa-Tomita S, Niihori T, Oba D, et al. New BRAF knock-in mice provide a pathogenetic mechanism of developmental defects and a therapeutic approach in cardio-facio-cutaneous syndrome. Hum Mol Genet 2014; 23: 6553-66.

\section{Update on the treatment of RASopathies}

Introduction. The term 'RASopathies' covers a series of diseases that present mutations in the genes that code for the proteins of the RAS/MAPK pathway. These diseases include neurofibromatosis type 1, Noonan syndrome, Legius syndrome, LEOPARD syndrome, Costello syndrome and cardiofaciocutaneous syndrome. Involvement of the RAS/MAPK pathway not only increases predisposition to develop tumours, but also determines the presence of phenotypic anomalies and alterations in learning processes.

Aim. To review the use of therapeutic strategies with mechanisms that have a selective action on RASopathies.

Development. The fact that the RAS pathway is involved in a third of all neoplasms has led to the development and study of different drugs at this level. Some of these pharmaceutical agents have been tested in RASopathies, mainly in neurofibromatosis type 1. Here we analyse the use of different antitarget treatments: drugs that act on the membrane receptors, such as tyrosine kinase inhibitors, in the mTOR pathway or MEK inhibitors. These latter have shown potential benefits in recent studies conducted on different RASopathies.

Conclusions. Today, thanks to the results from the first studies conducted with MEK inhibitor based mainly on animal models, a number of promising clinical trials are being carried out.

Key words. MEK inhibitor. mTOR. Plexiform neurofibroma. RAS/MAPK pathway. RASopathies. Treatment. 